This item was submitted to Loughborough's Institutional Repository (https://dspace.lboro.ac.uk/) by the author and is made available under the following Creative Commons Licence conditions.

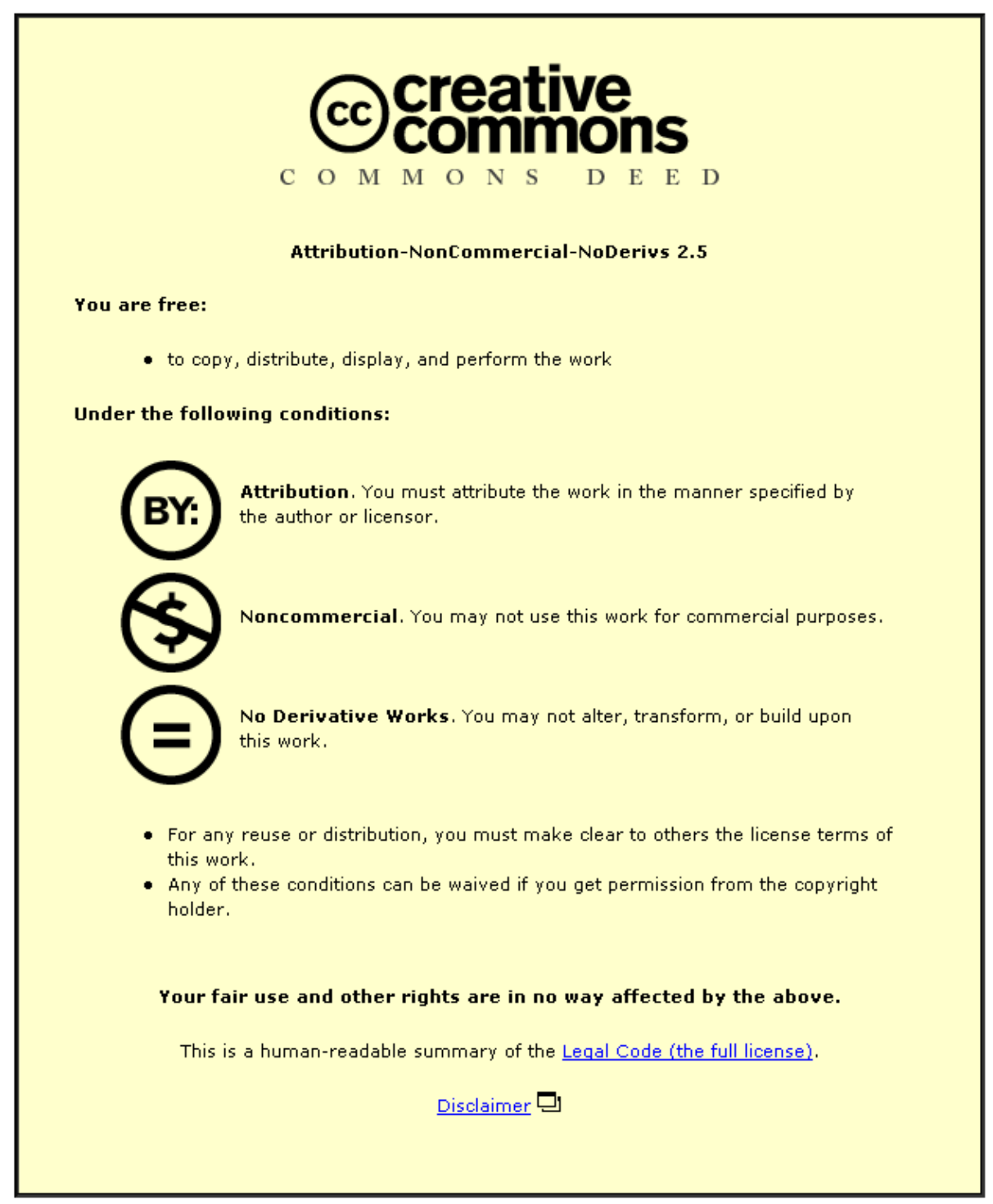

For the full text of this licence, please go to: http://creativecommons.org/licenses/by-nc-nd/2.5/ 


\title{
A Functional Architecture for an e-Engineering Hub
}

\author{
Z. Ren ${ }^{1}$, C. J. Anumba ${ }^{2}$, G. Augenbroe ${ }^{3}$, T. M. Hassan ${ }^{4}$
}

\begin{abstract}
Many Information Technology (IT) tools now exist to support collaborative working between engineering organisations. These often address operational issues rather than tactical or strategic issues. In particular, there are no effective tools for collaborative project planning between project partners. An electronic engineering hub (e-Hub) is considered useful in this regard. This paper presents the functional architecture of the eHub - a major research result of the EU funded e-HUBs project (e-Engineering enabled by Holonomic and Universal Broker Services). The e-HUBs project developed a universal collaboration platform for engineering outsourcing with focus on supporting collaborative project planning process. The e-Hub offers an extended set of functions to engineering service providers (mostly small and medium-sized enterprises) such as application hosting and definition and development of collaborative engineering workflows. The paper discusses the theoretical background and the supporting technologies for the development of the functional architecture and presents the deployment of the engineering e-Hub prototype in a construction scenario.
\end{abstract}

Keywords: collaborative project planning, e-Hub, e-engineering services, workflow

\section{INTRODUCTION}

Small and Medium-sized Enterprises (SMEs) are considered the backbone of the European economy, as well as a key source of jobs and a breeding ground for the knowledge-based economy. Flexible integration of resources and engineering collaboration with SMEs is expected to facilitate the strengthening of the competitive position of SMEs in their struggle for the future through acquisition of a critical mass in terms of necessary skills, capabilities and capacities. The e-HUBs project targets the conceptual development and implementation of a novel concept for the realisation of distant engineering collaboration of SMEs. The introduction of the e-Hub to broker and foster the agile formation of engineering partnerships lowers the barriers for the integration of SMEs into a dynamic global economy and creates opportunities for the formation of new SMEs [1].

Although collaborative virtual environments (CVE) hold major promise for the execution of collaborative engineering projects, the potential added-value, viability and practicality of these generic platforms have not been proven in the formation and execution of agile eEngineering partnerships [2]. The reasons are manifold, ranging from inappropriate implementation to missing theoretical and methodological foundations of the collaboration process. The e-HUBs project team started from the observation that lack of support for collaborative project preparation and planning was one of the important limitations of current partnership platforms. Below are the business and technical drivers for the study of collaborative project preparation and planning:

\footnotetext{
${ }^{1}$ Senior Lecturer, School of Technology, Glamorgan University, UK. Email: zren@glam.ac.uk

${ }^{2}$ Professor, Dept. of Architectural Eng., Pennsylvania State University, US. Email: anumba@engr.psu.edu

${ }^{3}$ Associate Professor, College of Arch., Georgia Institute of Tech., USA. Email: fried.augenbroe@arch.gatech.edu

${ }^{4}$ Senior Lecturer, Dept. of Civil and Building Eng., Loughborough University, UK. Email: t.hassan@lboro.ac.uk
} 
Business Drivers: Rather than betting on the "extended enterprise" formula, companies now express a desire to engage in on-the-fly partnerships. Ad-hoc partnering in project specific dynamic settings provides the agility that long term strategic alliances cannot guarantee. Efficient integration of engineering services on an ad-hoc basis into engineering projects is of strategic importance. These realisations have companies looking for support to initiate and plan partnerships that are remote, time-critical and volatile. Such partnerships necessitate a new generation of collaborative project planning methodologies and services. This is a key element for the effectiveness of dispersed collaborative engineering teams thus adding to the business value in collaborative engineering platforms. However it should be understood that these partnerships cannot rely on cultivated and documented business roles and trusted relationships as is customary in long-term alliances. On the contrary, in short term partnerships the rules of the project are one-off and need to be negotiated rapidly, transparently and explicitly. The delivery of generic project planning (PP) functionality is crucial in this respect, as this is the precursor to a whole range of other services that enhance the productivity and competitiveness of the partnership during execution of the work.

Technical Drivers: Three major facts have been observed in the relationship between outsourced work and the client's overall work schedule (Fig. 1):

1) PP inherits the earlier phases of the client project: "ad-hoc";

2) PP fuses the client and provider business processes; and

3) Typically, no effective methods are in place to manage and execute this in the client organisation.

The e-Hub platform development is driven by the need to offer the collaborative PP services that focus on collaborative, tactical decision making that goes into the formation, work planning, contracting and trust building on both sides of an e-engineering partnership.

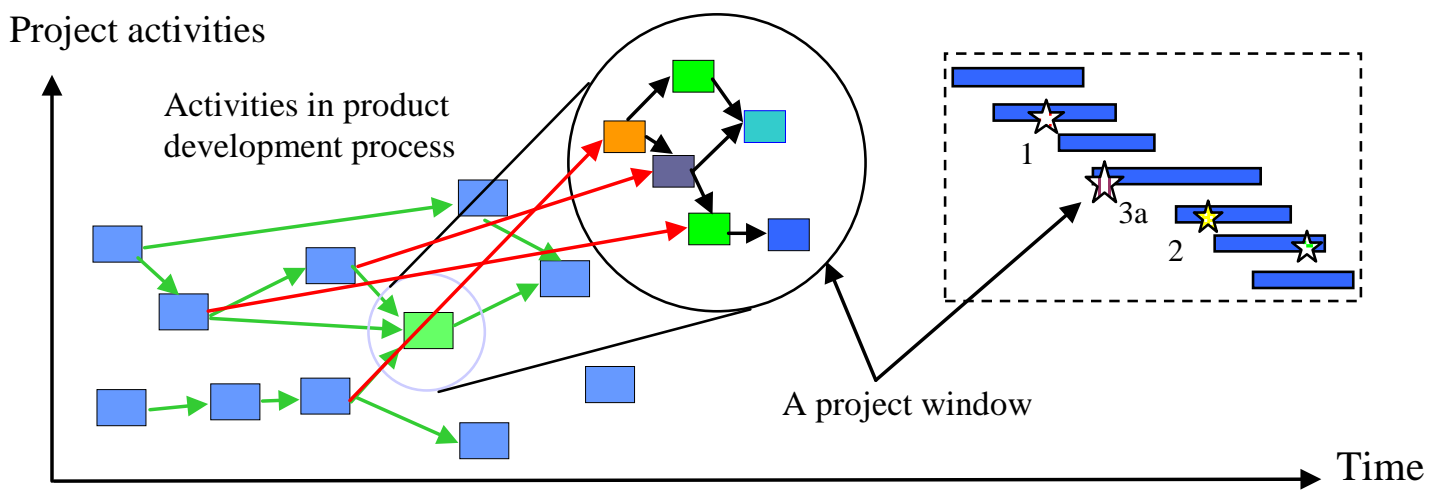

Fig. 1. Project activities in a defined project window [3]

The following sections present the key aspects of the development of the e-Hub's functional architecture. The joint development of a set of collaborative process templates and their configuration tools will be at the centre of the discussion. The term 'project' used in this study means the 'job' to be outsourced in a particular "project window" and the role of an e-Hub is to plan the execution of that job. Out of the five steps of online outsourcing (i.e. 1) deciding what to partner; 2) finding the right partner; 3) agreeing with that partner what, how, when to do it; 4) executing the plan; and 5) dissolving the partnership), the eHub focuses on Stage 3 with the understanding that this stage is under-developed, underrecognised and hence the biggest obstacle between potential partnering and actual partnering [4]. 
The e-Hub is novel in terms of the engineering services it offers (i.e. facilitating project definition and planning between volatile partners) and the functional architecture supporting such engineering services. This makes the e-Hub different from any other engineering collaboration platforms. Examples of these other platforms are: eHub_UK [5], which focuses on partner finding by considering the engineering expertise of each individual SME, and e-Builder [6] which offers dedicated collaboration services to architecture, construction and other industrial sectors. Other examples of CVEs include ToCEE [7], ICCI [8], KNOW-CONSTRUCT [9], NCCTP [10] all of which mainly facilitate the collaboration between different project participants during the project execution process.

\section{METHODOLOGY FOR PROJECT PLANNING SUPPORT}

Although PP is paramount for successful project management and execution, there is no generic collaborative PP methodology. Existing frameworks such as PMBOK [11] and PRINCE2 [12] are extendable to cover the collaborative dimension, as proven techniques and tools to support them are not available. It can be argued that the PP is to a certain extent deterministic. Moreover, for every domain, a client will be able to define a set of rules that express the logic of the PP process. This thinking has led to the formation of a project planning model (PPM), which contains the meta-rules of PP. It is assumed that every client will be able to define a PPM for a certain type of project. The PPM forms the central component from which a PP process is managed. An e-Hub that addresses the needs of many clients and many types of projects will contain different PPMs. This section reviews the essential PP issues and addresses the methodology for the e-Hub to support collaborative PP process.

\subsection{Project Planning: Content vs. Process}

According to the PMBOK, PP can be analysed from two basic views that lead to a deeper insight of PP, namely an object-oriented view and a process-oriented view. Two fundamental questions with regard to project planning are:

- What is the content of the resulting project plan and what are the constituent objects that need to be considered during the planning phase?

- What is the best procedure during the planning phase and what activities need to be executed in order to achieve the best planning result?

These questions are addressed below in the context of the e-Hub development.

\section{1) Content of Project Planning}

The objective of classic project preparation and planning is to define the project scope, timeframe, budget and other key issues of the project. With visible targets and constraints, PP turns into a set of subtasks coping with optimal resource allocation, control and management of issues related to various aspects of projects. At the preliminary planning stage, a pre-feasibility investigation is conducted, resulting in the pre-feasibility study such as expression of interest, draft of a business plan, analysis of technical feasibility, or potential drivers. At the detailed stage, an in-depth feasibility study and negotiation are conducted, detailed work plans are generated in terms of process quality assurance, finance management, process component interfacing, logistical optimisation, scheduling, and so forth (Fig. 2). 
Ideally PP should be structured, predictable and generic for all the types of projects. Not surprisingly many government institutions, agencies, and large corporations often have their own well-defined formal document templates to specify the content of project plans. These templates cover the generic content of each sub-plan. For example, Process Protocol [13], Project Management Template [14] and Project Planning Template [15] all provide IT supported PP templates. By following such project plan templates, users are able to address the key issues of project planning. It should be noted that these templates enforce rigour and governance, but have little to offer in terms of efficiency and multi-partner collaboration.

\begin{tabular}{|c|c|c|}
\hline $\begin{array}{l}\text { PP objectives } \\
\text { - description of the status quo } \\
\text { - definition of common goals } \\
\text { - definition of project scope / } \\
\text { delineation of tasks } \\
\text { - definition of boundary conditions } \\
\text { o time restrictions, } \\
\text { o budgets, } \\
\text { o organisational requirements, } \\
\text { o information input from other } \\
\text { - } \text { resource requirements } \\
\text { - tasks } \\
\text { - communication representations and } \\
\text { requirements }\end{array}$ & $\begin{array}{c}P P \\
\text { process/ } \\
\text { activities }\end{array}$ & $\begin{array}{l}\quad \text { Content of Project Plan } \\
\text { - } \text { project summary } \\
\text { - } \text { project charter and general scope statements } \\
\text { - } \text { project trade-off matrix \& status summary } \\
\text { - } \text { project organisation } \\
\text { - } \text { activity list/ work breakdown structure } \\
\text { - } \text { work product identification } \\
\text { - } \text { project schedule } \\
\text { - } \text { estimated cost at completion } \\
\text { - } \text { resource plan } \\
\text { - } \text { project requirements } \\
\text { - } \text { risk plan } \\
\text { - } \text { configuration management plan } \\
\text { - } \text { quality plan } \\
\text { - } \text { project communications and collaboration plan } \\
\text { - } \text { change control plan } \\
\text { - } \text { project procurement plan }\end{array}$ \\
\hline
\end{tabular}

Fig. 2. PP objectives vs. PP content

\section{2) Project Planning Processes}

PMBOK classifies PP processes as core processes and facilitating processes (Fig. 3). The core planning processes have clear inter-dependencies and thus require to be performed in essentially the same order in the majority of projects. The core planning processes may be iterated several times during any one phase of project planning. Iterations among the second class of planning processes, namely the facilitating processes, are dependent on the nature and structure of the project. 


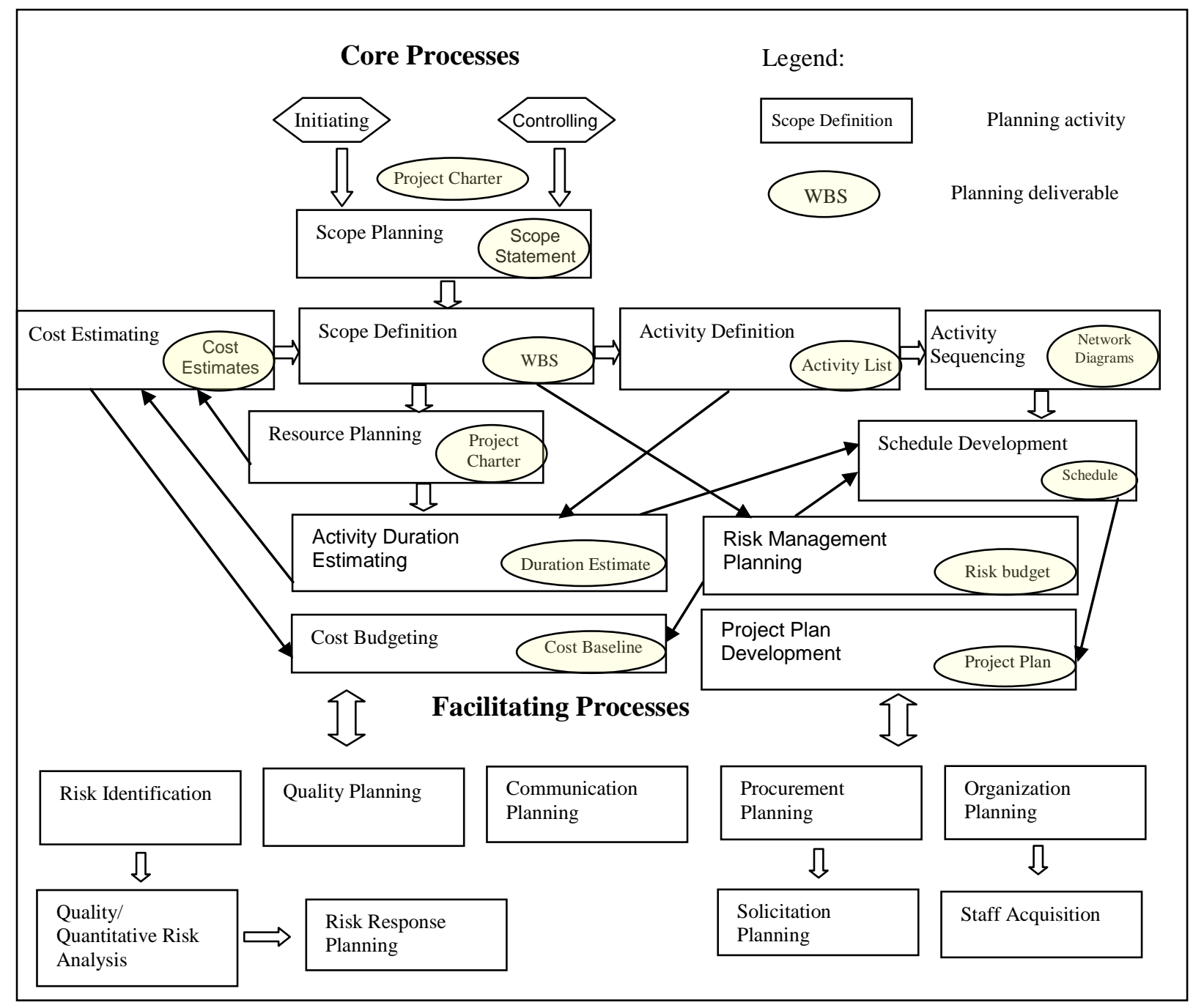

Fig. 3. Core Processes and Facilitating Processes (PMBOK)

\subsection{The Collaborative Aspect of PP}

Collaborative PP offers great opportunity for project participants to share and balance the client and provider's objectives, resources, expertise and constraints. This is fundamentally important for the success of agile engineering outsourcing. The e-Hub provides both general CVE functions (email, VOIP, fax and online meeting) and a set of dedicated collaboration approaches that are embedded in the PP process. The latter is associated with the role, content, process and result as formally defined in the PPM.

Collaborative PP generally has the form of an iterative loop, or of a dialog, in which the client's requirements and provider's proposals are continuously discussed and gradually refined. Clients usually have problems in the clarification of what is possible and what is desirable to expect from the project in return for invested resources, while providers want to balance available resources and expected efforts, associated with fulfilment of the client's requirements. PP is subject to frequent iterations prior to completing the project plan. A project plan, from the preliminary form to the final forms, is developed through all stages and refined from stage to stage. The PPM defines and controls (during enactment) what should be defined at which stage. 
The PPM is not one single model but a collection of models. Each of these models consists of a PP process model that incorporates the coordination logic of how the project planners negotiate and reach a resolution on one of the aspects that need to be agreed. Each of the workflow models operates on one or more content templates. A content template is an ordered set of fields with specific meaning.

\section{TECHNOLOGY}

Best of breed technologies for Internet-based communication, team collaboration and operational e-engineering form the baseline of the e-Hub development. They are positioned at the core of the e-Hubs. The specific information technologies deployed for the PP extensions are workflow management system and generic CVE.

\subsection{Workflow Management System for Process Management}

WfMC [16] defines workflow as: "The automation of a business process, in whole or part, during which documents, information or tasks are passed from one participant to another for action, according to a set of procedural rules”. Gerogakopoulos et al. [17] give a more explicit description of a workflow process. They define a workflow as a collection of activities organized to accomplish some business goals. An activity can be performed by software system(s), human(s), or a combination of these. In addition to a collection of activities, a workflow may include constraints that influence the order of performing activities as well as information flow between them.

The essential workflow characteristics are roles, activities, application tools and resources [18]. The roles perform tasks using application tools that provide access to various shared information resources. Marshak [19, 20] defines the "3Rs" and the "3Ps" of workflow technology as follows:

Rules: Workflow systems take various business rules into account.

Routes: A route is strongly coupled to the concept of information logistic that typically supports organisation flows of all kinds of objects including documents, forms and processes.

Roles: Information is routed to roles rather than to a particular person. The role in an organisation is a group of people with the required skills and authority.

Processes: Business/engineering processes span over organisation units and legacy information systems.

Policies: Policies correspond to a normative process model that describes how certain processes should be handled.

Practices: This is the way in which work is actually performed in the organisation.

Workflow Management System (WfMS) provides computer-based support for the task of workflow management. It supports the specification, execution, and dynamic control of workflows involving humans and information systems [21]. Fig. 4 shows an outline of a WfMS architecture. The process analysis, modelling and definition tools facilitate the specification of the components of a workflow as a process definition. The workflow enactment service enacts a process definition by assigning tasks to humans and software systems while also maintaining the constraints between tasks. The workflow control data represents the dynamic state of the workflow system and its process instance, which is managed and accessible by the workflow management system. The workflow relevant data is used by the workflow management system to determine the state transitions of the workflow instance. 


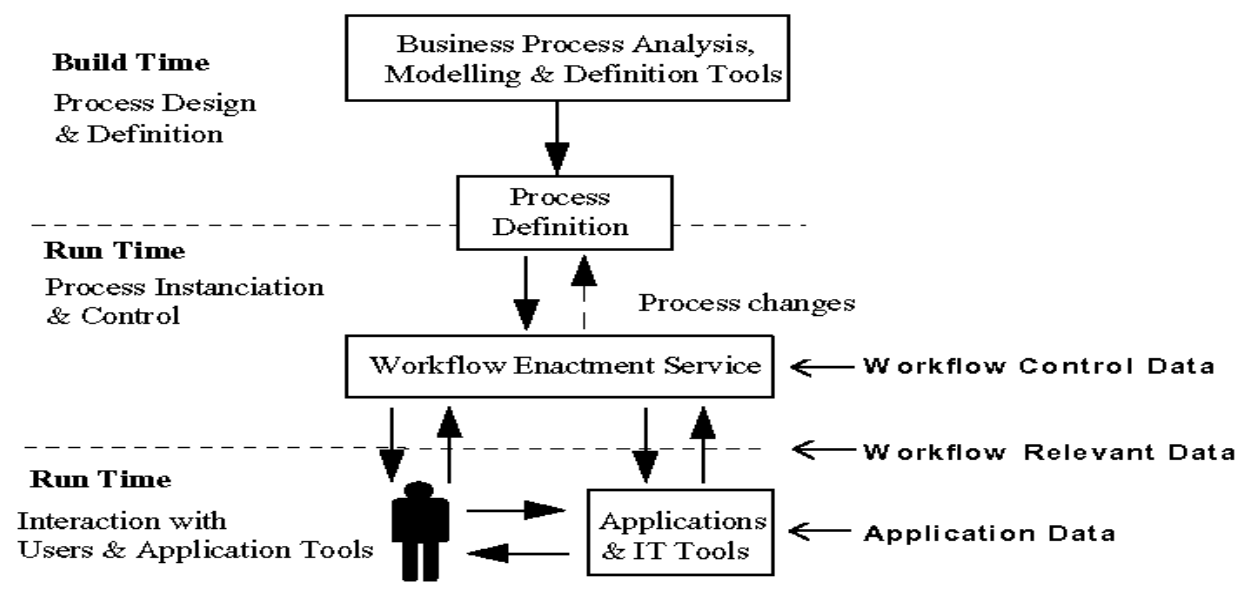

Fig. 4. Workflow system architecture and data structure [16]

The central PPM consists of a set of WfM that codifies the logic of the activities that partners engage in during project planning. As such, the PPM enforces that the collaborative PP is conducted in a way that is consistent with the rules that are (implicitly) expressed in the PPM. This is in line with the views expressed by Prior [22] who summarises the three major solutions which a WfMS provides to business and engineering problems:

- a unique and systematic approach to model business or engineering processes where the key features involved in the business or engineering processes such as roles, activities, inter-dependencies, routes and resources are considered;

- an effective tool to administer contract management; and

- an approach to facilitating knowledge management, records management and process monitoring.

The e-Hub’s workflow management services include:

- A graphical Process Modeller for designing workflows, the modeller is a configuration of the Java Workflow Editor, JAWE [23]. Workflows are represented in the XPDL (XML Process Definition Language) format, compliant with the WfMC standards.

- A Workflow Module for the enactment of workflows. It imports XPDL files created by the Process Modeller and enacts the workflows. When initiating the PP, users can utilize the Workflow Module to gain access to all project related information. Users are automatically notified of actions they are requested to complete (as defined in the workflows being executed), while all produced documents are routed to designated activities and roles.

\subsection{Technologies for Collaboration}

To facilitate collaboration, a Basic Collaboration Platform (BCP) has been developed in the e-HUBs project, built on Java and J2EE technology. The BCP is a best of breed CVE that provides two levels of services: common services and engineering services. Some of the key features of the common services include:

- User Management: registration, building user profile, access controls via groups/roles, directory;

- Collaboration Features: email, forum, online chatting, newsgroups, discussion groups, calendar and meeting support; 
- Document management: upload and download, access right, version control, copy right, and Enterprise Applications Integration (EAI);

- Internationalisation: portal support for different character sets, portal support for different date formats and time zones;

- Security: encryption of key data as required, access control, restricting access to data and documents, cross-authentication or single-sign-on mechanisms;

- Others: browser compatibility, Graphical User Interface (GUI), searching, access to Web services, mobile/wireless computing, openness.

In the BCP a set of activity components are added to support specific needs. Among them are a PP whiteboard, annotation function and a specific contract editor as examples of embedded external services.

- The PP Whiteboard allows users discussing a particular section of the project plan online both textually and graphically. Users can import screenshots from planning documents or use a drawing toolbox to create sketches, use online chatting and drawing to discuss the details, and save the animated discussions.

- The annotation function allows users to mark and explain changes on existing standard documents. This is helpful to make the e-Hub a transparent and traceable environment for joint work plan definition and negotiation.

- The BCP is also able to support and integrate some external services such as eLEGAL contract editor (CE) [24] and Ganttproject [25]. Although such services are not technically integrated as an e-Hub service, the logical binding between the e-Hub and $\mathrm{CE}$ is achieved through the use of customisable workflows.

\section{FUNCTIONAL ARCHITECTURE}

The functional architecture of the e-Hub, which harmonises the group collaboration functionality provided by the BCP with the dedicated PP functional components, is described in this section. Collaborative PP is viewed by the e-Hub as a managed collaborative process that transparently generates a comprehensive "project plan", consisting of a set of interlinked documents. Documents are either structured models or unstructured (text) information. The added value of the e-Hub is that the generation process is composed of logically ordered activities that drive the collaborative generation of (partly) structured content. The logical ordering of activities and their relationship to structured content is embodied in a formal PPM as explained above. The PPM is a set of workflow models, each of which operates on one or more content templates.

Fig. 5 shows the functional architecture for this. It shows a workflow model which specifies that a task is to be assigned to a particular project planner (a member of the project group), while three fields of the information template have been defined to be accessible by this task. At the time of enactment, the project planner that is assigned to this task, will complete these fields with specific information. In doing so, she/he may use links to internal documents and external resources whenever appropriate, as indicated in Fig. 5. It is very important that workflows can be started at will and run concurrently, reflecting the multi-tasking and multi-threading working styles of real life project planning. Planners typically work on different issues concurrently (Fig. 6). 


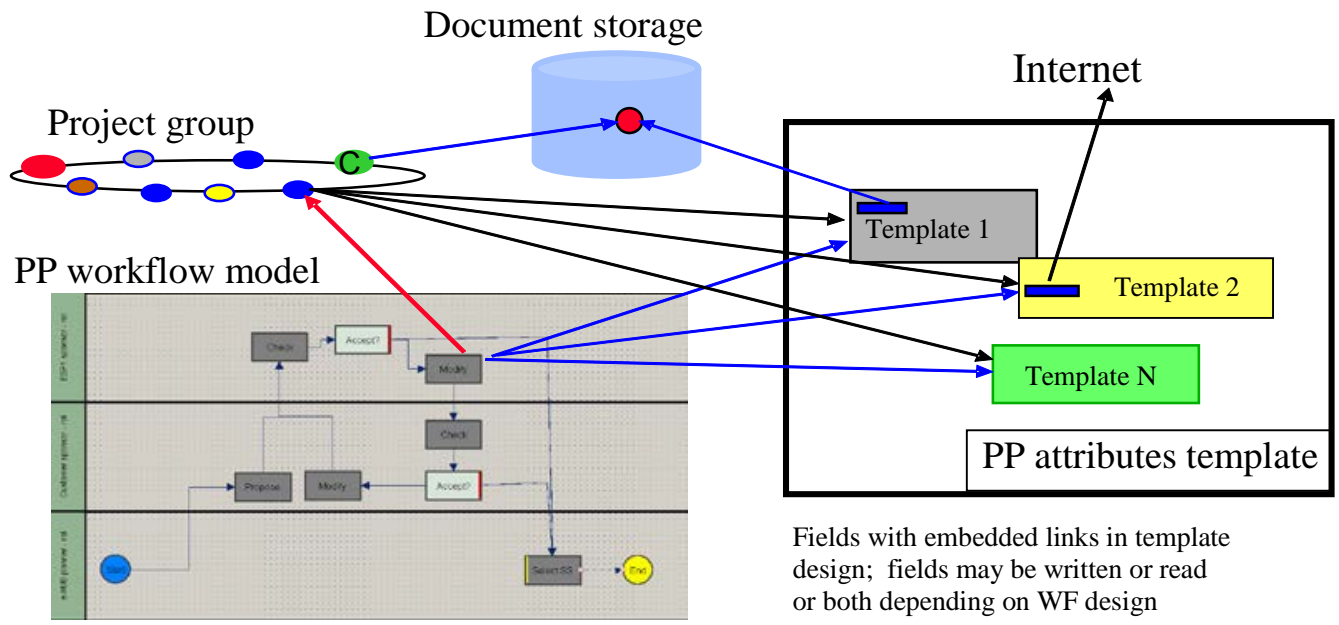

Project team member

PP template

PP workflow activity

C PP coordinator

Data storage

Fig. 5. Workflow models and linked information templates

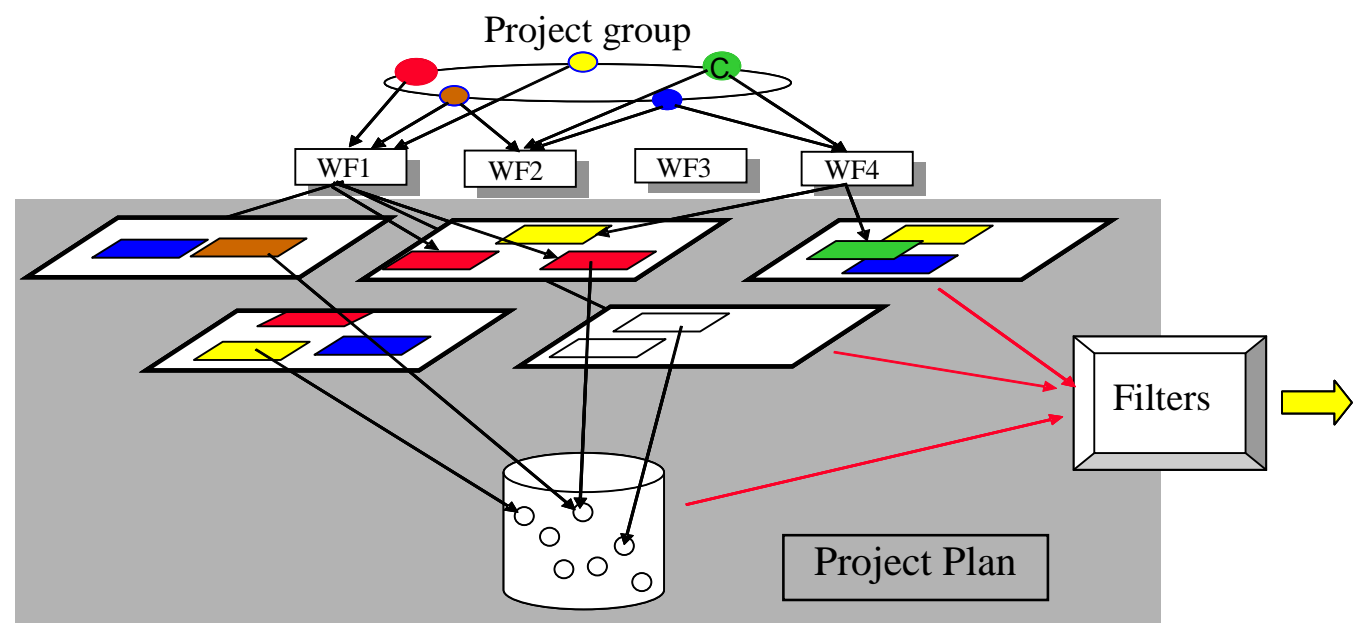

Project team member

C PP coordinator

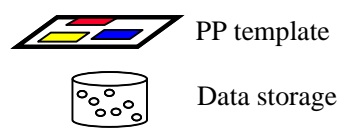

Fig. 6. Workflows operating on a collection of templates

Fig. 6 shows the typical situation where a project planning team deploys a PPM with four workflows. In this case three workflows have been executed, or are executing concurrently (WF1, WF2, WF4). WF3 has been uploaded in the workspace but has not been executed, whereas WF2 is executing but has not yet reached the state where interaction with information templates has taken place.

In the figure it is assumed that the four workflows have been defined to interact with five different information templates. Each template has a set of fields and different members of the project team have inserted information in these fields as mandated by the logic in the workflows (the coloured fields). It is also indicated that fields may contain hyperlinks to stored documents and other fields thus making the space of project documents (the Project Plan) a rich information resource which can be navigated in many different ways. It is now 
possible to define filters in the e-Hub workspace that transform the generated project information into structured reports, contract templates, and project execution schedules.

Fig. 7 shows a high level architecture of the e-Hub distinguishing the five major functional modules and how they operate on communities (containing client, providers, external consultants etc), documents, workflow logic and templates.

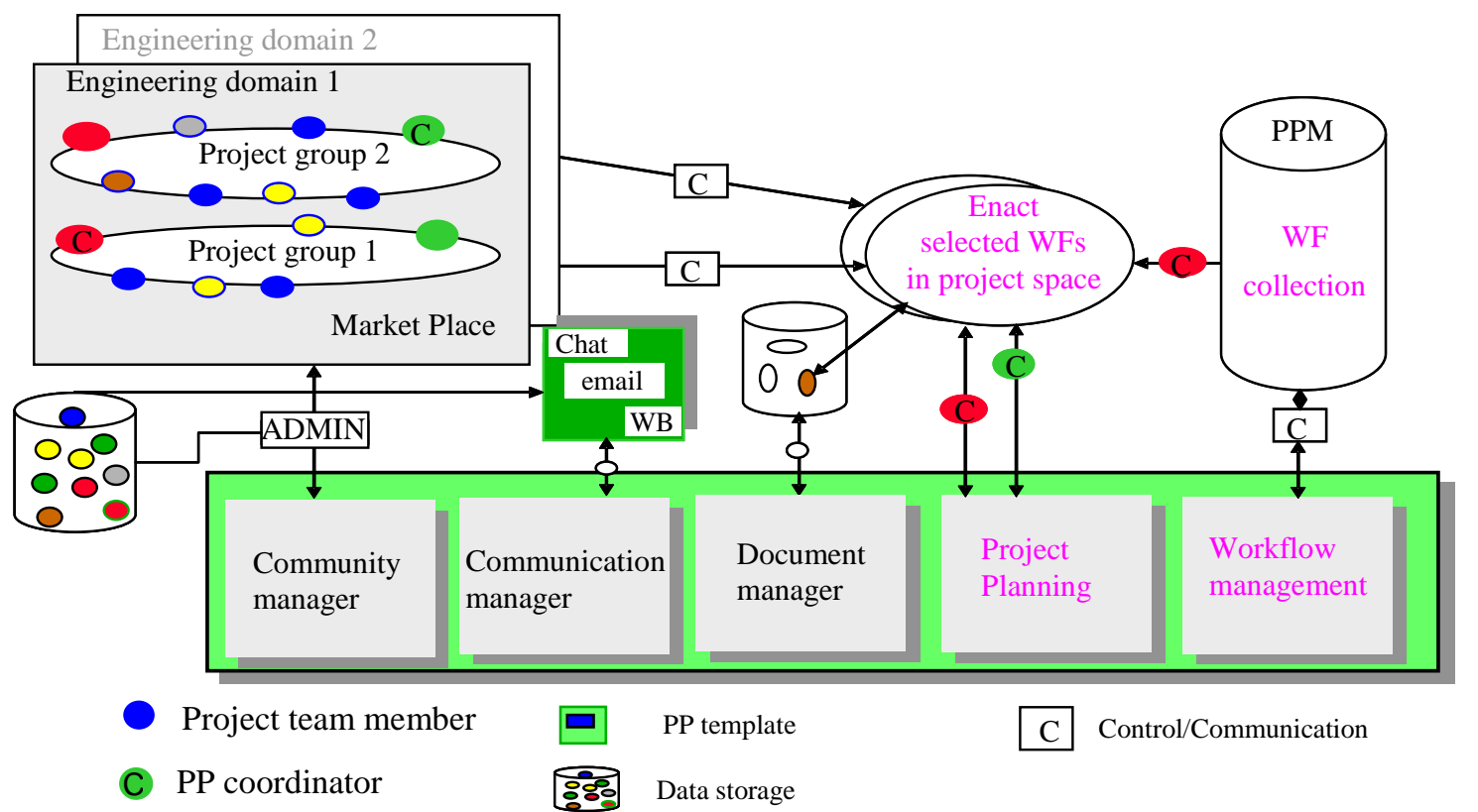

Fig. 7. Functional Architecture of the e-Hub

The figure also shows how the community manager module (managed by an administrator) maintains the identities of all users, and assigns access rights to engineering domains and any active project within the engineering domains (managed by a domain Coordinator). Every engineering domain has an open Market Place, to which every registered domain user has automatic access.

The communication manager module controls chat, email, web meeting and other events, whereas the document manager provides access to document. The workflow management module stores and manages the PPM, creates instances of workflows and exports instances to the WF run time environment. Coordinators of project groups decide which PPM, or which packages within a PPM will be made available to the project team. The project planning module enacts an instance of a workflow, allows the assignment of actual project partners to roles defined in the WF, maintains the link between WF variables and native eHub applications, and manages the link between WFs and information templates.

The above architecture combines the functions for community control, process control and managed generation of information in the predefined templates. It is this mix that enables a structured project planning dialogue (i.e. arrive at a joint resolution about tasks, responsibilities, dependencies, incentives, penalties during project design etc.).

It is one of the longer term objectives of PP communities to harness the commonality of project planning steps and store them as PPM templates. The e-Hub business entity could act as the custodian of these templates and make them available to new PP teams in the same domain. If the PPM templates contain proprietary client knowledge it is more likely that the client will retain the intellectual property and will make models only available to 
internal project teams. In either case, the e-Hub business entity will offer services (either public or for specific clients) that allow the development, extension and testing of PPM templates.

\section{APPLICATION IN SEISMIC SERVICES SCENARIO}

The developed functional architecture has been tested in construction and manufacturing scenarios. These SME-driven scenarios demonstrated how SMEs can use the e-Hub to perform and contract a client's non-core engineering work. One of the scenarios deals with an existing firm - Geodeco - a consulting company based in Italy specialised in geotechnical, geo-environmental and earthquake engineering. It currently provides these services through a Web Portal (eRiskZone, [26]). In this case, it is assumed that Geodeco will use the e-Hub to offer engineering services to a Dutch design firm (the Client) who is seeking advice on seismic risk assessment.

Three key planning issues have been identified by project participants in the seismic engineering scenario: initial project brief and cost estimate, project execution plan/schedule definition and contract negotiation. Accordingly, three workflows and related attribute templates have been defined with each representing a key phase of the project planning process. Fig. 8 illustrates the PPM developed for this scenario.

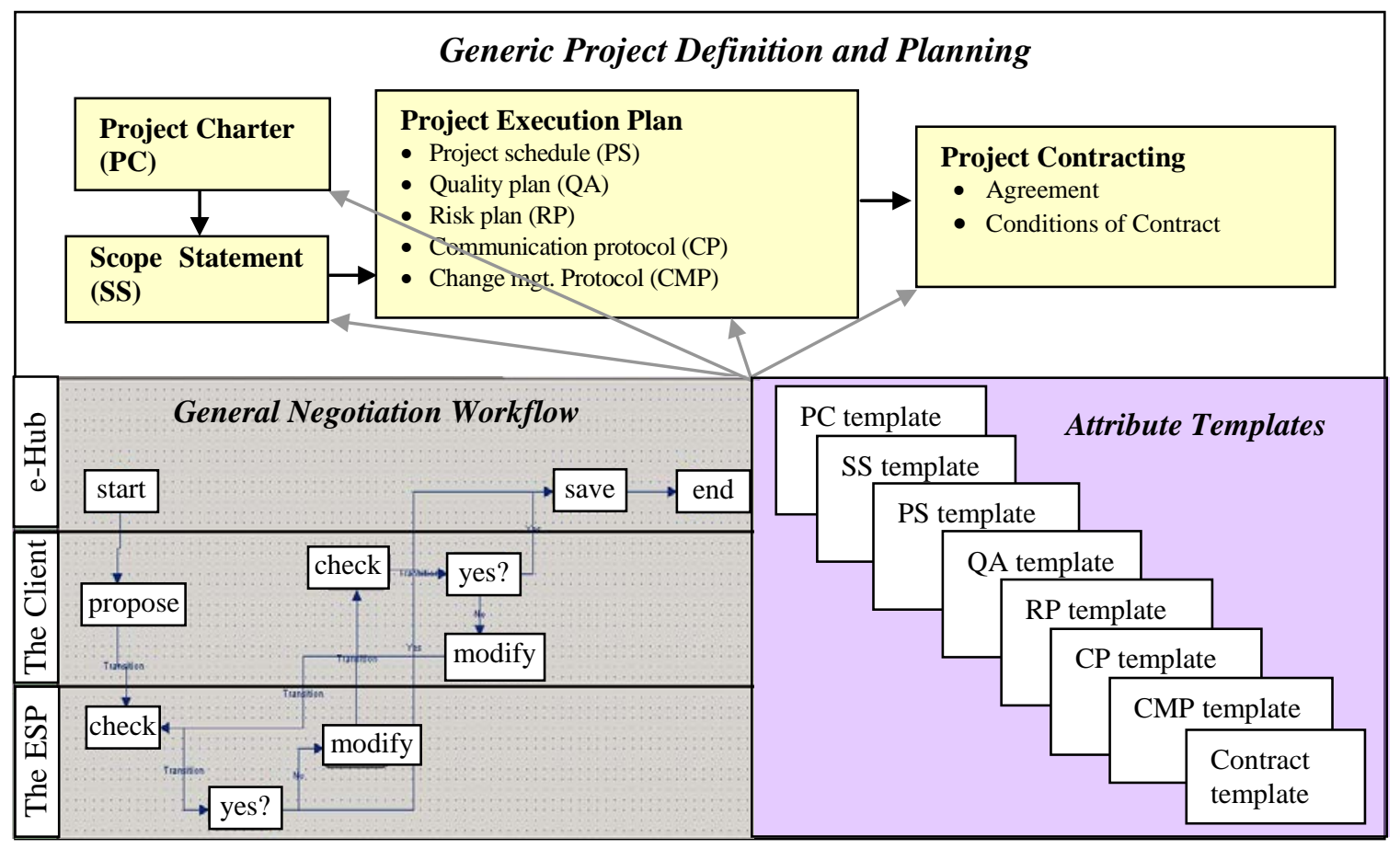

Fig. 8. Generic project definition and planning workflow embedded in the e-Hub

\section{Workflow 1: Project Definition and Cost Estimate Workflow}

This workflow defines the process of preliminary project definition and initial project cost estimate. This process extends the project participants' hand-shaking process initiated in Geodeco's eRiskZone portal [26]. After preliminary discussions in the portal, the project participants switch to the e-Hub to further define the project and negotiate the most important element for cooperation (i.e. the cost for service). According to the Client and Geodeco, this workflow includes the following activities:

- Define the basic project charter and scope;

- Define project input requirements; 
- Address project output requirements;

- Specify any particular requirements including methodology, material, equipment, or other resources;

- Negotiate project cost.

Fig. 9 illustrates this collaborative project definition process. A generic negotiation workflow is embedded in this workflow which allows users to negotiate each project definition information item. The figure also lists the attributes to be negotiated in the negotiation workflow. These attributes have been derived from a structured elicitation of the Client's and Geodeco's industrial experiences. This elicitation process drives the development of the PPM and information templates.

\section{Workflow 2: Project Execution Plan and Scheduling Workflow}

After both parties reach an agreement on the essential project definition and initial cost estimate, they, together with other partners, enter a stage to define various detailed project work statements. These work statements address the most important collaborative issues for the execution of the project (e.g. project schedule, quality plan, risk plan, change protocol and communication protocol).

In the chosen scenario, the project schedule was particularly important for the Client, therefore the related workflow and attribute template are fully developed and implemented in the scenario. To define the project schedule, a number of activities will typically be performed by project participants including:

- Define activities;

- Identify dependencies;

- Define duration;

- Specify deliverables;

- Address milestone.

\section{Workflow 3: Contract Negotiation Workflow}

After defining the collaborative work plans, the Client and Geodeco (as well as other participants) enter the contract negotiation stage. An agreement template is developed based on standard engineering service outsourcing contracts. A contract negotiation workflow was developed to facilitate the negotiation of the key contract items in the agreement template. For example, there are four key items (i.e. no. of test samples, final service cost, liquidated damage, and governing laws) to be addressed in the agreement template, the contract negotiation workflow will guide the Client and Geodeco to negotiate these items step by step. The items finally agreed by both parties will fill into the 'right place' in the agreement template which (in this case) is saved in the eRiskZone database. The particular advantages of e-Hub mediated project planning for this scenario are:

- The agreement template highlights the key contractual issues for each particular engineering service outsourcing situation so that users can finalise all the key issues before they sign the contract.

- The collaborative work statements generated through previous workflows are integrated into the agreement template, which provides a sound basis for the service outsourcing contract. 


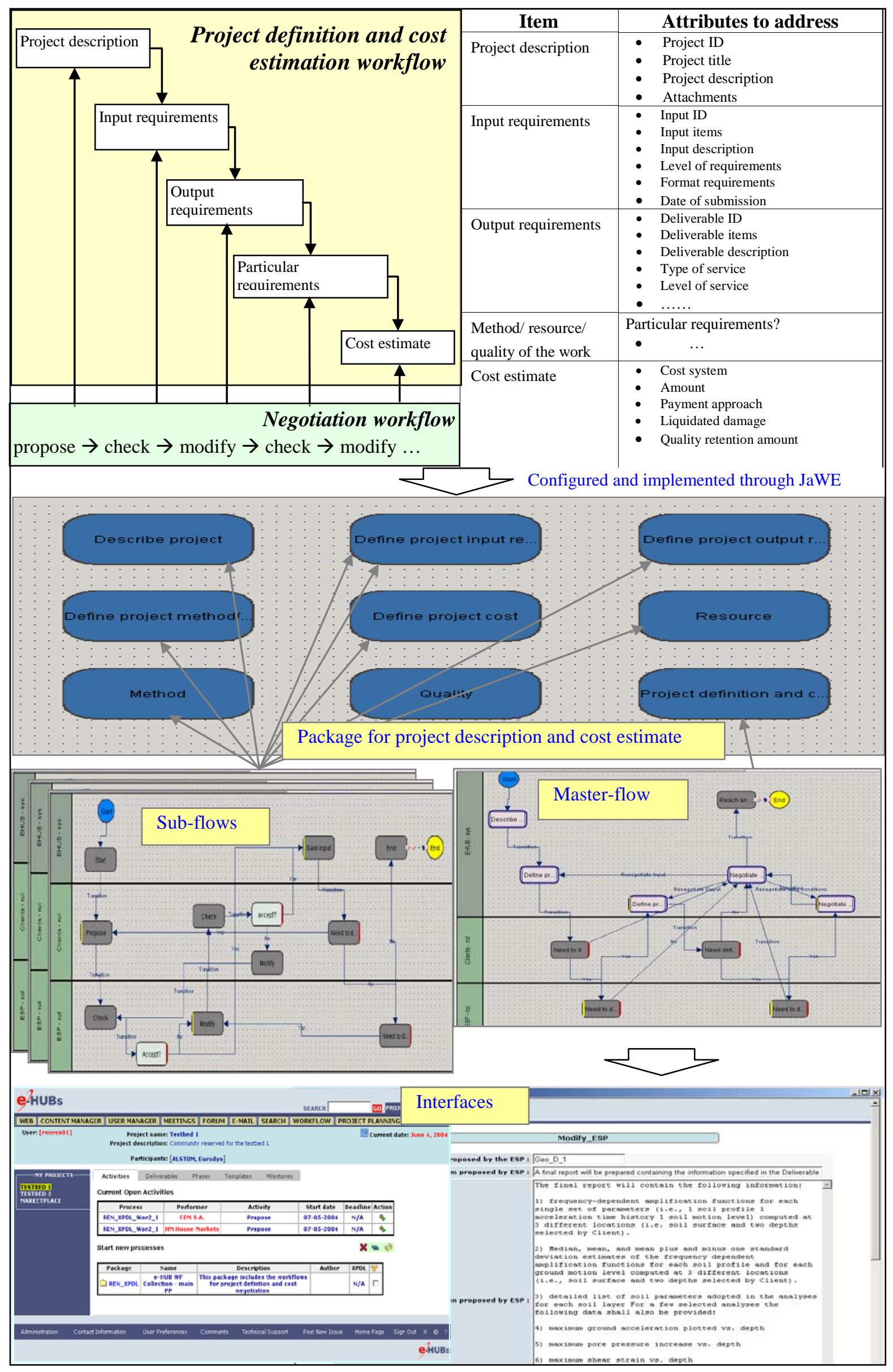

Fig. 9. Project description and cost estimation WF 
It is important to note that workflows may require certain engineering support services to be implemented in, or deployed in co-existence with, the e-Hub. In this case these services include:

- Workflow 1: To estimate the service cost, project participants need cost estimating tools. In this case, Geodeco adopts a spreadsheet to estimate the service cost based on five key factors (i.e. scope of service, methodology, data provided by the client, time, and overhead). By defining these inputs and the related parameters, project participants estimate the initial project cost by using the cost estimation tool. These input factors are addressed by the project definition workflow. The outcome from the workflow will be input to the cost estimation tool.

- Workflow 2: Although the workflow specifies the process of defining the project schedule, particular engineering tools are necessary for project participants to undertake detailed scheduling. Companies normally have their own scheduling tools such as MS Project, Primavera, Bar charts and GanttProject. The integration of these tools allows users to combine them with the dedicated functions of the e-Hub. Users are able to access the scheduling tools during the enactment process of workflows. In this scenario, GanttProject [16] is adopted as the scheduling platform.

- Workflow 3: The eLEGAL CE provides a comprehensive contract negotiation platform for the e-Hub. Contract negotiations conducted on the CE are tightly integrated with the eHub workflow. Guided by the contract negotiation workflow, the CE provides both offline and online contract negotiation platforms. In this scenario, the CE is mainly used to negotiate the Conditions of Contract, which often involves complex contract clauses to be specified in workflows. A Conditions of Contract template for engineering services has been developed, which is stored in the eRiskZone database and linked with the CE.

\section{EVALUATION OF PROTOTYES}

The e-Hub prototypes [27] have been evaluated using various approaches: internal evaluation by the development team, expert clinics, virtual interviews and online demonstrators [28, 29, 30]. Four key issues of the system (i.e. fitness, usability, utility and business impact) have been assessed. The results show that users are very positive about the fitness, utility and business impacts of the e-Hub while the usability is ranked rather low. This suggests that online help and initial training are necessary in order to use the e-Hub prototype effectively and efficiently.

It should also be recognised that the low usability of the e-Hub prototype is not only an interface problem; it also reflects the immaturity of the overall e-Hub concept. Further development of the e-Hub should consider the integration of the collaborative project planning with the project execution process. Currently, the e-Hub infrastructure has been adopted in the SEEM (Single Electronic European Market [31]). As a novel concept, the engineering e-Hub needs to be implemented in more cases so that the concept could be testified and further improved.

\section{CONCLUSIONS}

As large companies get leaner, the opportunities for dedicated SMEs are growing, but geographic boundaries remain a bottleneck for SMEs to expand their services. The e-Hub concept is a response to this in that a Web hosted e-Engineering broker removes distance as a constraint in collaborative engineering. In the SME-dominated construction industry, this 
is expected to lead to a rapid uptake of ICT technologies by SMEs. Surveys have shown that the cost of technology-heavy e-business solutions is the biggest barrier for smaller companies to enter the global e-business community [2]. The e-Hub concept removes this barrier and adds economy of scale to globally operating SMEs.

Supported by a set of dedicated engineering Web-Services, the developed e-Hub's functional architecture provides users with a unique workspace to conduct project planning and contract negotiation. Users are able to plan the details of the work to be outsourced collaboratively and negotiate the contract with the support of the generic PP workflow and attribute templates. The functional architecture makes an innovative contribution to the facilitation of collaborative planning. The scenarios have shown that the e-Hub has the potential to grow into a universal broker that facilitates the engineering outsourcing work in different industries.

Business opportunities for the type of e-Hubs introduced in this paper seem abundant as they hold promise to innovate the marketplace of ASP based e-services in the majority of the following aspects:

- Offer job procurement, contracting and collaborative process facilities, including handshaking, process sharing and process mediation;

- Offer low entry level access for SMEs to the global marketplace for the outsourcing and fulfilment of engineering subtasks;

- Offer configurable e-Engineering process templates, thus harnessing proven procedures for remote collaboration;

- Provide a trusted engineering gateway to SMEs;

- Develop collaboration methodologies in a specific sector and identify business champions;

- Support new organisational development in e-collaboration;

- Consolidation of best practices, exemplified in qualify assurance procedures and certification of SMEs; and

- Facilitate international trade agreements in affiliation with (local) trade and industry sector organisations.

The materialisation of these expectations will require further development and testing, in particular in industry-strength e-engineering scenarios. These tests and other follow-ups should address the potential mismatches between PPM formality with the informal culture of project planning, completeness of information, acceptability and usability by project planners and others.

\section{ACKNOWLEDGEMENTS}

The e-Hubs project was supported by the European Commission under the IST programme (Contract no: IST-2001-34031). The authors would like to acknowledge the financial support of the European Commission, and record their appreciation to all the e-Hubs project partners.

\section{REFERENCES}

[1] G. Augenbroe, e-HUBs: e-Engineering enabled by Holonomic and Universal Broker services, eAdoption and the Knowledge Economy: Issues, Applications, Case studies. P. Cunningham and M. Cunningham (Ed.), IOS Press (2004) 1285-1292. 
[2] A. Shevchenko, H. Horváth, and J.S.M. Vergeest, Formal Requirements Specification for e-HUBs. e-HUBs Project Deliverable D1, 2002.

[3] G. Augenbroe, e-HUBs “Level-1” Work Plan 2003.

[4] e-HUBs, Project Technical Annex-1. e-Engineering enabled by Holonomic and Universal Broker Services, Description of Work. IST project: (IST-2001-34031), 2001.

[5] J. Bal, and N. Armoutis, eChallenges for Manufacturing Regions and Their SMEs, A Case Study: The West Midlands Collaborative Commerce Marketplace, In the proceedings of Innovation and the Knowledge Economy: Issues, Applications, Case Studies, (Ed.) P. Cunningham, and M. Cunningham, IOS press, ISBN 1-58603-563-0, Ljublijana, Slovenia, 2005.

[6] e-Builder: http://www.ebuilder.net/, last accessed on $14^{\text {th }}$ Feb., 2008.

[7] ToCEE (Towards a Concurrent Engineering Environment in Building and Engineering Structures Industry): http://cic.vtt.fi/projects/tocee/index.html, last accessed 03/2007.

[8] ICCI (Innovation Co-ordination, Transfer and Deployment through Networked Cooperation in the Construction Industry, IST-2001-33022): http://cic.vtt.fi/projects/icci/public.html, last accessed 03/2007.

[9] KNOW-CONSTRUCT (Internet Platform for Knowledge-based Customer Needs Management and Collaboration among SMEs in Construction Industry, COLL-CT-2004500276): http://www.know-construct.com/index.htm, last accessed 03/2007.

[10] NCCTP (Network for Construction Collaboration Technology Providers): http://www.ncctp.net/about.htm, last accessed 03/2007.

[11] Project Management Institute, A Guide to the Project Management Body of Knowledge (PMBOK), Newtown Square, PA, 2000.

[12] C. Bentley, PRINCE2 - A Practical Handbook, Oxford: Elsevier-Butterworth Heinemann, 2005.

[13] G. Aouad, J. Hinks, R. Cooper, D. M. Sheath, M. Kagioglou, M. Sexto, An IT Map for a Generic Design and Construction Process protocol, Journal of Construction Procurement, 4(1) (1998) 132-151.

[14] Project Management Planning Template, http://www.dof.ca.gov/OTROS/StatewideIT/SIMM/SIMM200/PM3.11\%20Planning\%20Pl an\%20Format.pdf\#search=\%22project\%20planning\%20template\%20\%22, (1997), last accessed 01/03/2006.

[15] Project Planning Template, http://www.michigan.gov/dit/0,1607,7-139-18391_2201658009--,00.html, last accessed 01/03/2006.

[16] WfMC, The WfMC Glossary. Workflow Handbook 2001, Future Strategies Inc., 2000, ISBN 0-9703509-0-2, pp311-352. 
[17] D. Gerogakopoulos, M. Hornick, and A. Shet, An Overview of Workflow Management: Form Process Modelling to Workflow Automation Infrastructure, Distributed and Parallel Databases, 3(2) (1995) 119-153.

[18] P. Jarvis, Workflow Literature Survey: The State of the Art in Workflow Systems. Artificial Intelligence Applications Institute, the University of Edinburgh, 1998.

[19] R.T., Marshak, Workflow White Paper: An Overview of Workflow Software, In the Proceedings of WORKFLOW’94 Conference, San Jose, 1994.

[20] R.T., Marshak, InConcert Workflow: Independent from XSOFT, InConcert Inc. Provides Flexible Workflow Underlying Engineering Team Support, Workgroup Computing Report, Patricia Seybold Group, 1997.

[21] D.R. McCarthy, S.K. Sarin, Workflow and Transaction in InConcert, Bulletin of the Technical Committee on Data Engineering, 16 N2 - IEEE, Special Issues on Workflow Extended Transaction Systems, 1993.

[22] C. Prior, Workflow and Process Management, Workflow Handbook, L. Fischer (Ed.) Florida, Future Strategies Inc., (2003) 17-25.

[23] http://www.enhydra.org/workflow/jawe/index.html, last accessed on 21st Jan., 2005.

[24] http://cic.vtt.fi/projects/elegal/public.html, last accessed on $21^{\text {st }}$ Jan., 2005.

[25] http://ganttproject.sourceforge.net/, last accessed on 21 $1^{\text {st }}$ Jan., 2005.

[26] http://www.geodeco.it/, last accessed on $14^{\text {th }}$ Feb., 2008.

[27] http://elf.eurodyn.com:8080/edos/index.do, last accessed on $21^{\text {st }}$ Jan., 2005.

[28] P. Crehan, D2.1: Business Development Plan for e-Hub Phase 1. e-HUBs Project Deliverable. IST project: (IST-2001-34031) (2003).

[29] P. Crehan, D2.2: Business Development Plan for e-Hub Phase 2. e-HUBs Project Deliverable. IST project: (IST-2001-34031) (2004).

[30] E. Baessler, R. Mejía, A.V. Grop, P. Crehan, and Z. Ren, D6.3: Evaluation Approach and Benchmark Results. e-HUBs Project Deliverable. IST project: (IST-2001-34031) (2004).

[31] http://www.seemseed.net/default.aspx , last accessed on $14^{\text {th }}$ Feb., 2008. 